\title{
The Problems with Immersive Advertising: In AR/VR, Nobody Knows You Are an Ad
}

\author{
Brittan Heller and Avi Bar-Zeev
}

Imagine five years from now, you're walking down a street wearing your own mixed reality glasses. They're sleek, comfortable and increasingly fashionable. A virtual car drives by-it's no coincidence that it's the exact model you've been saving for. Your level of interest is noted. A hipster passes on the sidewalk wearing some limited-edition sneakers. Given your excitement, a prompt to buy "copy \#93/100" shows up nearby. You jump at the chance, despite the hefty price. They'll be waiting for you when you get home.

Cinema and television have long been imagining what advertising will look like in XR (known alternatively as eXtended Reality and as the entire Mixed Reality continuum from Virtual Reality (VR) to Augmented reality (AR)). We're reaching the point where science fiction is rapidly becoming reality.

If you've watched professional sports on TV in the past decade, you've almost certainly experienced a form of augmented advertising. Your friend watching the same game across the country will likely see different ads-not just the commercials but the actual billboards in the stadium behind the players may be replaced to suit the local advertising market.

VR-based advertising is in its infancy, and it looks very different from traditional advertising because of the way immersive media works. Instead of simple product placement, think about immersive ads as placement within the product. The advertising is experiential, using characteristics of media and entertainment that came before, alongside embodiment (a feeling of physical presence in a virtual space) and full immersion into a digital world. In immersive environments, creators completely determine what is seen, heard, and experienced by the user. This is not just influencing your feelings and impulses, but placing you in a controlled environment that your brain will interpret as real.

As advertising in immersive contexts takes off, we should do more than marvel at the pokemon we meet in the street. AR and VR experiences have profound effects on cognition that are different from how we take in and process information in other media. For example, our brains interpret an assault in a VR world, ${ }^{1}$ even in a cartoonish environment, just like we are being attacked in our own homes. These implications get even more complex when we consider paid content: how will the unique characteristics of immersive worlds be used to persuade and drive behaviors?

Further, XR systems can collect a staggering amount of different types of data from XR users, from mechanisms like eye tracking and gesture-based controls. This is fundamental to the hardware, so users may not have a choice (or even realize what is happening). On top of that, XR systems have the potential to use data to serve even more precisely

1. https://carrcenter.hks.harvard.edu/publications/reimagining-reality-human-rights-and-immersivetechnology 
targeted advertising-not just on demographics, but on deeply emotional or physiological responses. Much scholarship, including fields like behavioral economics, has emerged around human decision making, specifically aimed at helping the marketing and sales world. Because of the way XR interacts with your brain, this type of advertising may require even more careful consideration.

In this paper, we first review the history of advertising in emerging media formats, and then consider the evolution of immersive environments so that we can build on what we know about these technological fora. In doing so, we will argue that the form of XR advertising matters just as much as the content, and that consumers and regulators should start weighing the benefits and detriments of advertising in XR today.

\section{The Move Toward The Metaverse}

Advertising exists in every medium, and as XR environments become commercialized and popular, it is inevitable that advertising will follow. Facebook's acquisition of Oculus in 2012, and the business's evolution into Facebook Reality Labs (FRL) in 2020, only accelerated this trend. ${ }^{2}$ This speculation was fueled by FRL's leader (now CTO), Andrew Bosworth, who previously ran Facebook's ad business and quipped in 2015 "that experience should include ads, because life includes ads." 3 He refused to disavow advertising as part of Facebook's immersive computing bet, as the company moved toward The Metaverse. ${ }^{4}$

The point of advertising in Oculus $\mathrm{VR}^{5}$ was described by the company as:

...[T]o bring more people into VR, advance the consumer experience, and make progress on our longer-term augmented reality initiatives. We're also exploring new ways for developers to generate revenue-this is a key part of ensuring we're creating a self-sustaining platform that can support a variety of business models that unlock new types of content and audiences. It also helps us continue to make innovative AR/VR hardware more accessible to more people.

This statement makes the same argument the company was making with social media: advertising will keep the platform "free" or "low cost." Of course, someone always pays. And in this case, the money comes from higher prices for the products we all buy. With this as a founding principle for one of the chief drivers of XR innovation, it should be no surprise that ads in the medium are in our future.

But advertising in immersive technology like VR and AR is not actually a new concept. Marketers know that immersive content is the most engaging type of media, ${ }^{6}$ which makes it a natural fit for advertisements. But like any immersive medium, the name of the game in advertising is often making your ads blend in, not stand out. The medium is following the well-trod path of other internet-based technologies and gaming, where the most effective ads are often indistinguishable from ordinary gameplay.

The way advertising has evolved in gaming and online platforms - from the earliest instances to product placement to interactive experiences ${ }^{7}$-has shaped how it manifests in AR and VR. There are still many unexplored areas. But the key question is not the degree of audience acceptance, tolerance or rejection of novel advertising, in whatever

2. https://techcrunch.com/2021/06/16/facebook-will-begin-beaming-advertisements-into-virtual-reality/

3. https://money.cnn.com/2015/09/22/technology/facebook-messenger-disrupt/index.html

4. https://about.fb.com/news/2021/09/building-the-metaverse-responsibly/

5. https://www.oculus.com/blog/testing-in-headset-vr-ads/

6. https://techcrunch.com/2021/07/08/can-advertising-scale-in-vr/

7. https://adsider.com/from-sega-to-vr-a-brief-history-of-ads-in-video-games/ 
form it takes. Rather the public needs to be aware of what potential harms may occur, individually and collectively, through the systematic collection, refinement, and experiential application of their deeply personal data, perhaps without their full knowledge and in ways that undermine their interests.

\section{Advertising in Movies and Sports}

Any review of advertising designed to identify the influences on AR and VR ads does well to start in cinema. The first motion pictures were the immersive media of their day, with stories about early silent films like the Lumière brothers' L'Arrivée d'un train à La Ciotat ${ }^{8}$ (1896), giving you the view of a train coming into a station as if you were on the platform, resulting in terrified audiences. It is debatable whether or not there was actually panic in the cinema, but in the alternative, writers of the day talking about the train crashing into the audience could have been describing the emotional power and convincing $3 \mathrm{D}$ impressions of the new medium of film.

Product placement-the granddaddy of embedded advertising-was first seen in movies in the 1940s. Product placement is a means to target audiences in both an indirect and engaging way. Whether it is E.T. eating Reese's Pieces or James Bond driving the BMW Z3 Roadster, ${ }^{9}$ seeing products depicted on-screen has been shown to influence consumers' purchase preferences. The main limitation is that it's been difficult to tailor the ads to the specific audiences, given how movies are made and distributed.

Everyone remembers the myriad billboard ads from stadiums they went to as a kid. But if you watched almost any professional sports in the last ten years, the ads you saw were most likely not the same as the ads in the physical stadium. Broadcasters track the spatial orientation of TV cameras and replace the ads with virtual alternatives, even with players walking in front. Local content distributors will often customize these ads based on local needs, in addition to any interstitial commercials they use to replace the broadcast feed. This technology is more overtly used to put virtual "first down" lines on the playing field in American football.

Professional athletes have long leased advertising space on their jerseys and vehicles. But they are beginning to lend their entire likeness for something called "digital twins" to hawk virtually any product, today or in the future. A "digital twin" is a life-like copy of a person, place, or thing, reproduced within a computer system. For people, these twins are often coupled with limited artificial intelligence to reflect the original personality, to the extent possible.

\section{History of Advertising in Platforms and Gaming}

But $\mathrm{XR}$ is at its heart an interactive experience, and examining how advertising evolved in previous interactive media can give us insight into where immersive advertising is headed.

Advertising in video games began with static ads, embedded into a game's code that always showed up in the same way. The first recorded advertising in a video game ${ }^{10}$ was in 1978, when Adventureland included a promotion for its creator's next game, Pirate Adventure. In the 1980s, non-gaming companies began to sponsor games in order to

\footnotetext{
8. https://www.atlasobscura.com/articles/did-a-silent-film-about-a-train-really-cause-audiences-tostampede

9. https://books.google.com/books?hl=en\&lr=\&id=LOTAV4BRs60C\&oi=fnd\&pg=PR9\&dq=advertising+in+ video+games+wolfenstein\&ots=Tl62bquI_3\&sig=A0Q2iYR4iHxU3W1ey0EtMNR_tkg

10. https://www.linkedin.com/pulse/essential-introduction-in-game-advertising-louis-duenas
} 
advertise their products. One such game by Coca-Cola was called Pepsi Invaders. ${ }^{11}$ This had the same dynamic as the popular Space Invaders, but the enemy ships spelled out "Pepsi."

In-game advertising emerged in 1984 with a game called Micro Olympics ${ }^{12}$ including ads paid for by tech brands, with company names displayed on the sides of the track. This set off a wave of product placement, like Chupa Chups lollipops and their logos forming the backdrop of the 1992 release Zool: Ninja of the Nth Dimension, ${ }^{13}$ conspicuously integrated advertising within gameplay. As the interactivity of console-based games evolved, passive placement of promotions transitioned to tying actual products, like specific models of cell phones, Doritos tortilla chips and Kentucky Fried Chicken, ${ }^{14}$ to the fundamental mechanics of gameplay.

With the onset of the world wide web, advertising began to appear around, as opposed to just within, games. Examples of this include the first banner $\mathrm{ad}^{15}$ in a web browser, deployed in 1994 by AT\&T, or ads running before a game begins like the previews for a film. Similarly, the technological shift to mobile gaming created new possibilities and form factors for games with the phone-based interface.

Mobile gaming brought about two relevant changes versus traditional console and PC games. The first was that mobile games tended to be more casual, played intermittently for shorter spans, like while commuting or watching television. The second was that the most common price for downloadable apps went from about one dollar to free in the race for customers. A good example is Angry Birds, ${ }^{16}$ which debuted at $\$ 0.99$ and sold over 2.4 million copies during its first year. Today, version 2.0 of the app is free to install, but with in-app purchases for some content.

To generate profit, most mobile game developers moved to this free-to-play model with both advertising and downloadable content deployed for late-bound monetization, meaning these games generally allowed totally free-play up front, but with limitations and opportunities to spend money later. For example, customers are enticed to buy "coins" to accelerate their "coin dozer" game ${ }^{17}$ instead of waiting for daily timers to slowly grant more. Game companies value "whales" 18 who they've reported as individually spending hundreds to thousands of dollars per game, effectively subsidizing many more casual players.

As mobile advertising developed, you can begin to see more and more signs of experimentation with "interactive" ads-advertising that is integrated into gameplay itself. For example, as an alternative to buying their way past daily timers that limit gameplay, gamers could watch ads to earn credits more quickly-an early step to blur the line between ads and gameplay. This tactic ties ads into the mechanics of the player's gaming experience, albeit in a crude way, but may be at the root of ads-as-experiences as embraced in XR.

Another step was the rise of "playable" ads-advertisements that are effectively games themselves, often embedded within other games or floating on websites to grab attention.

11. http://www.atarimania.com/game-atari-2600-vcs-pepsi-invaders_11875.html

12. https://medium.com/etermax-brand-gamification/in-game-advertising-four-decades-of-innovation$9 \mathrm{a} 2 \mathrm{~b} 3 \mathrm{f} 7 \mathrm{c} 75 \mathrm{c0}$

13. https://www.cracked.com/article_20879_the-5-least-subtle-product-placements-in-gaminghistory.html

14. https://www.cracked.com/article_20879_the-5-least-subtle-product-placements-in-gaminghistory.html

15. https://www.8thwall.com/blog/post/41172588959/advertising-enters-the-next-dimension

16. https://www.wsj.com/articles/BL-SOURCEB-6606

17. https://play.google.com/store/apps/details?id=com.leftover.CoinDozer\&hl=en_US\&gl=US

18. https://venturebeat.com/2013/03/14/whales-and-why-social-gamers-are-just-gamers/ 
Game companies place a second advertising-game within a popular game. ${ }^{19}$ Players are asked to play for a few minutes and then are invited to download the new game before moving on with the current one. This experience combines the notion of ads-asroadblocks with the emerging technique of ads-as-experience itself.

Across all of these experiences, a careful review identifies numerous dark patterns ${ }^{20}$ (user interface design built to channel users to preferred outcomes) at work. Mechanisms for a user to "close" ads or embedded games are often hidden, obscured or badly designed. Games may insist the player rate the game (five stars suggested) very early on.

In a different pattern, many free apps secretly harvest location-based information for data brokers ${ }^{21}$ to better target ads outside the game. The most popular of these has been Angry Birds. Mobile phone companies like Apple and Google attempt to combat ${ }^{22}$ these practices ${ }^{23}$ with app store reviews and rejections, but many concerns remain. ${ }^{24}$ Some phone carriers, however, may have directly engaged in such data sales. ${ }^{25}$

It's estimated that at least $2 \%{ }^{26}$ of apps on the iOS app store today are misleading or fraudulent.

\section{Advertising and the Pressure of Do Not Track}

But online advertising is not a runaway train. The industry is changing amidst pressures for increased consumer privacy and calls for regulation. For example, the industry has long relied on "last click attribution" that attempts to predict behavior based on the last thing a consumer did (before buying a product or using an online service). However, that's not how people actually behave. Buyers often take time to think about purchases, do research, and revisit later. Accordingly, Google is moving to data-driven attribution, ${ }^{27}$ that models a person's activities overall and, while this may be more accurate it also leverages more personal data.

Apple is also changing how other companies approach advertising. Previously, companies could track people across mobile and web experiences. Often, all it took was embedding a 1-pixel graphic in an email or webpage, reporting back to a central database when accessed. But more sophisticated analytics could determine whatever users spend time on, what apps they install, games they play, and music they listen to.

Apple is ramping up ${ }^{28}$ enforcement of "do not track" policies, reducing the internal features of browsers to hide uniquely identifying information, requiring more disclosures for apps that collect information, adding protections from emails that scrape information, anonymizing identity (Hide my Email, Sign-in with Apple, Apple Pay), and even adding VPN-like services to iCloud for more private web browsing. Apple is even limiting what it knows via services like Find My Phone or Air Tags, by moving more Siri speech recognition to your local device instead of sending audio to the cloud.

19. https://www.gamesindustry.biz/articles/2015-10-09-zynga-launches-gamified-ads

20. https://uxdesign.cc/game-design-dark-patterns-that-keep-you-hooked-a3988395533c

21. https://www.nytimes.com/interactive/2019/12/19/opinion/location-tracking-cell-phone.html

22. https://developer.apple.com/app-store/review/guidelines/

23. https://www.washingtonpost.com/technology/2021/06/06/apple-app-store-scams-fraud/

24. https://spectrum.ieee.org/new-tool-strips-manipulative-dark-patterns-from-mobile-apps

25. https://www.washingtonpost.com/technology/2019/01/10/phone-companies-are-selling-your-locationdata-now-some-lawmakers-want-federal-investigation/

26. https://www.aarp.org/home-family/personal-technology/info-2021/warning-signs-of-fraudulentapps.html

27. https://blog.google/products/ads-commerce/data-driven-attribution-new-default/

28. https://www.apple.com/newsroom/2021/06/apple-advances-its-privacy-leadership-with-ios-15ipados-15-macos-monterey-and-watchos-8/ 
Overt advertising or tracking isn't the only game that advertisers may play. Newspapers wrestled with the ethics of whole-page ads that were written to look like journalism. Google initially limited advertising to small text ads on the edge of the search results, but then eventually moved to paid placement with its ad-words system.

These developments lead to questions about what sponsored content will look like in $X R$. Will the rise of do not track, which will depress the value of advertising on phones and in other spaces where Apple's and other's efforts are having the most impact, drive advertisers toward VR, where these efforts do not yet apply?

\section{Social Media Advertising}

This brings us to the most fertile proving ground for advertising techniques that will migrate into XR: social media. Social media is a flat "virtual world" that many users inhabit for hours every day. They interact with flat "avatars" (in this case, icons) of their friends, consume content, and play games. It may not be fully immersive, but it's the clearest non-spatial alternative to a persistent, immersive experience-particularly for advertising.

Today, social media inserts ads right into people's timelines or feeds, alongside organic content from friends and family. Some may argue social media intentionally blurs the line between paid and unpaid posts, and creates a sense of false intimacy. In the case of Twitter, people can "like" and comment on these ads just like other tweets, with only a small "promoted" message at the bottom to warn us of the sponsored source. Most recently, we see individual people with followings also posting such promoted content. On all platforms, paid posters seek to build up substantial followings and then promote their messages. While these branded voices can often be clever and entertaining, ${ }^{29}$ engagement is the ultimate point.

Influencers are people who have intentionally built up massive social media audiences, often by aggregating and commenting on other people's content. Some influencers, like top Twitch streamers, ${ }^{30}$ TikTok stars, ${ }^{31}$ and YouTubers, ${ }^{32}$ make seven or eight figure incomes from endorsements and media contracts. Some are simply enthusiastic consumers, like kids who unbox toys. Overall, influencers are like sports commentators, creating more excitement for the game. Followers may or may not trust an influencer's recommendations for all products, but the influencers add enough attention that at least some followers will follow through on their recommendations.

What's even more remarkable is that influencers need not be human anymore. Lil Michaela is an entirely computer-generated human ${ }^{33}$ run by BRUD, a startup. The FTC has guidelines that require influencers to make clear when posts are sponsored. ${ }^{34}$ But it's not clear if this is being enforced with digital humans.

29. https://www.ranker.com/list/the-best-of-wendys-twitter/mick-jacobs

30. https://twitter.com/KnowSOmething/status/1445663228831297545

31. https://www.ravereviews.org/entertainment/tiktoks-top-earners/

32. https://www.huffpost.com/entry/how-much-influencers-make___5dee68a6e4b05d1e8a556bbc

33. https://www.forbes.com/sites/mattklein/2020/11/17/the-problematic-fakery-of-lil-miquela-explainedan-exploration-of-virtual-influencers-and-realness/

34. https://www.ftc.gov/tips-advice/business-center/guidance/disclosures-101-social-media-influencers 


\section{Immersive Advertising: the Perfection of the Advertising "Experience"}

On May 17, 2021, FRL announced they would be piloting ads in Oculus VR. ${ }^{35}$ This was shortly followed by an announcement, on June 17, 2021, that ads in-headset in Oculus VR would begin in a game ${ }^{36}$ called Blaston VR by Revolution Games. But within five days, Blaston VR pulled out of the trial run, ${ }^{37}$ after a severe backlash from its user base.

However, that doesn't mean that immersive experiences and games do not already have forms of advertising. For our purposes, VR includes any immersive 3D world a person may temporarily inhabit, whether it's on a computer screen or head-mounted display.

Traditional forms of advertising can be found in VR, like billboards within a virtual world. ${ }^{38}$ Product placement and sponsorship for experiences are also quite common, because VR content is still quite expensive to produce. Influencers and celebrities put on events, just like they would in physical reality, and you can buy virtual luxury-branded accessories for your digital avatar using your real money. Brands are interested in the greater interactivity and dynamicity of VR, because it can achieve a higher level of audience engagement. ${ }^{39}$

Due to this, more frequently, immersive advertising takes the form of experiences, sometimes crossing multiple forms of media that users voluntarily enter into for entertainment. For example, there are several cinematic VR experiences and games that place you in the heart of Jurassic Park, ${ }^{40}$ allowing you to interact with dinosaurs-including one featuring Blue, a velociraptor who is a main character in Jurassic World. Various games are playable for home-based VR users with their own head mounted devices, to bespoke hardware you can purchase to use VR coming from your phone, to location-based experiences ${ }^{41}$ you can play with a friend at Dave and Buster's arcades. As with advertising in other interactive experiences like games, VR developers are focused hard on blurring the line between advertising and the experiences ads promote. In fact, VR games and experiences are often advertisements themselves. What better way to make someone excited to see the next Jurassic Park film than to place them inside the adventure themselves?

Other advertising experiences can include special events in VR, such as concerts by top-label talent, ${ }^{42}$ or watching sports games as if you had the best seat in the house. These are experiences (some that users even pay for), but they are also ways to promote an artist, a team, a VR platform or a brand, without having your consumers leave the comfort of their own home. Thus musicians can continue to perform virtually during a pandemic without traveling, or even keep touring long after they are dead. ${ }^{43}$

Finally, VR should be considered a porous medium. With web-based VR, or the ability to access the internet via a VR headset, any advertising you can find on the internet can potentially hit users in VR.

35. https://www.oculus.com/blog/see-yourself-in-vr-with-live-overlay-plus-new-infinite-office-featuresand-more-in-latest-oculus-update/

36. https://www.oculus.com/blog/testing-in-headset-vr-ads/

37. https://www.eurogamer.net/articles/2021-06-23-oculus-developer-pulls-out-of-facebook-in-headsetvr-ads-trial

38. https://www.tandfonline.com/doi/full/10.1080/15252019.2020.1846642

39. https://www.tandfonline.com/doi/abs/10.1080/15252019.2019.1596850?journalCode $=$ ujia20

40. https://www.vrse-vr.com/product/details/jurassic-vrse

41. https://reflexarc.co.uk/projects/jurassic-world-vr

42. https://www.rollingstone.com/pro/features/virtual-reality-livestreams-covid-1021683/

43. https://www.washingtonpost.com/magazine/2019/10/30/dead-musicians-are-taking-stage-againhologram-form-is-this-kind-encore-we-really-want/ 


\section{How AR Integrates Ads}

The biggest innovations in mobile advertising are found in AR, which we define as an experience that combines everyday immersion in the real world with computer-generated elements added. Today, AR combines phone cameras, screens and special algorithms (like Apple's ARKit ${ }^{44}$ or Google's ARCore ${ }^{45}$ ) to make the phone seem like it's adding onto the real world. Common examples include Instagram filters and Snap Lenses.

In the near future, wearables, like AR goggles, smart glasses, and someday even contact lenses ${ }^{46}$ will modify a user's perception of reality with superimposed 3D objects. There are several early entrants in the "race to your face" for wearable glasses (see NReal, Magic Leap, HoloLens), but thus far these are expensive and not well-featured for consumer use. Facebook's Ray-Ban Stories smart sunglasses are more affordable and fashionable, but do not have AR visual displays in the initial release.

Packaging and promotional materials can blend with AR, bringing more life to advertising. The simplest use case is a 2D movie poster or physical product, such as a branded bottle, box or can, to experience a fun and occasionally interactive "pop up" 3D experience on top. The movie poster pops out of the wall with animated 3D content or video with compelling audio, grabbing and holding our attention for far longer than any traditional movie poster ever did. The wine company "19 Crimes" similarly augmented their wine bottles ${ }^{47}$ with 3D experiences existing within the labels, animating and storytelling the various “criminals" in their brand. While Snap doesn't pay its Lens creators yet, some lenses have billions of unique downloads already. The creators can make money today from marketers by creating custom Lenses that hawk a brand, for example by augmenting products, people's faces or bodies, with interesting visuals, ${ }^{48}$ like turning someone's face into a talking Taco Bell taco.

People tend to think of AR as being largely geospatial, but this kind of content can be triggered anywhere on the designated objects. QR codes or products themselves can be used to engage AR content. The trend now is for more physical products to come with a digital layer that extends the experience using phones.

We can observe more sophisticated applications from companies like Ikea ${ }^{49}$ and Nike ${ }^{50}$ that help sell their products directly, by combining AR with e-commerce. ${ }^{51}$ Ikea lets you place virtual 3D furniture in your actual home using AR to see how it might fit and complement your existing walls and furniture. Once you're happy, you hit a button and order directly (assembly still required). Nike lets you look at virtual sneakers placed over your actual feet, enticing you to virtually try on and then buy from an expensive line of sneakers without going to the store. These use cases are already mediating the way people shop through their technology.

Marketers widely expect visual search to work for AR, as Google's Lens app does today, where users walk down the street, and see apparel or other products in the store window or on even the street, worn by people passing by. Your phone or glasses may display what the product is, how much it costs, where else it can be purchased, and if possible, provide a link to purchase instantly.

\footnotetext{
44. https://developer.apple.com/augmented-reality/arkit/

45. https://developers.google.com/ar/

46. https://www.mojo.vision/

47. https://tactic.studio/19-crimes-ar/

48. https://www.adweek.com/performance-marketing/taco-bells-cinco-de-mayo-snapchat-lens-wasviewed-224-million-times-171390/

49. https://www.architectmagazine.com/technology/ikea-launches-augmented-reality-application

50. https://www.marketingdive.com/news/nike-kicks-up-coloring-book-with-ar-sneakers/581454

51. https://www.syte.ai/blog/visual-ai/vr-ar-and-visual-search-changing-the-way-we-shop/
} 


\section{What is Coming Next?}

Targeted advertising across all visual media may be on the horizon. It is not yet common for your personal household television set to replace ads in a deeply personalized way, the way your social media feed is targeted. But if your TV or connected device (e.g., Apple TV) can play 3D games, it is already theoretically possible.

When we consider XR devices, we don't expect only virtual 3D billboards or interstitial commercials. We expect to see more experiential 3D product placement, integral to storytelling. So, for example, if a story calls for a car, a particular sponsor's car will be introduced for the player to drive. Any object could be replaced based on hidden automatic ad auctions.

The missing piece is access to a database of ad classifiers on users, like Facebook has amassed, or like databases that are available for sale from data aggregators. The goal is not to make ads that one can't skip (like the controversial TiVo feature to fast forward through commercials), but to make ads that the recipient does not want to skip or may not even realize are ads, as inseparable from experiences. We see the level of craft that goes into Superbowl TV ads every year. And indeed some people watch these just for the entertainment value, ignoring the sports.

If ads were to use more personal information, and most importantly, known emotional triggers, to entice the viewer to watch and absorb the content, they would become effectively irresistible. For example, a popular Duracell TV commercial ${ }^{52}$ featured a military dad missing his daughter and sending her a toy. The ad was selling batteries, but appealed to most parents' love for their kids. It could have been even more targeted to appeal to military spouses (or people who really need batteries). Parents will generally relate to ads featuring a range of emotions around raising children (love, frustration, fear). Teenagers will respond to emotions of belonging or social "value." Ads using virtual actors that physically or behaviorally resemble the people one most cares about (including oneself) will also become more compelling. These features can soon be custom-tailored to the individual emotional triggers and the social network of the target.

Market research and adaptive advertising may be conducted on live XR users, by swapping products and styles in and out of the scene in rapid succession. Research ${ }^{53}$ has demonstrated that people will not notice certain kinds of environmental content changes during ordinary blinks, or while their eyes are moving or focused elsewhere. People who attempt to memorize their surroundings to defeat such a system may be surprised to learn the system may be aware of their current focus and change something else instead. A person's broad-based preferences for other people (including sexual attraction), products, places, politics, and more will be determined in mere hours or minutes instead of years-and often based on involuntary physical responses to external stimuli. And the results of this information may be used to further optimize products placed into view, for maximum likeability and persuasion.

\section{Risks and Harms}

The discussion of harms stemming from digital advertising often begins with the question of people's tolerance of ads. We will occasionally layer in privacy debates over whether people's information is truly "sold" or merely "misused." Future experiential and immersive advertising will be so subtle, and perhaps even enjoyable, as to feel like an integral part of the user experience. Tolerance or likeability of advertising is therefore the wrong

52. https://www.youtube.com/watch?v=DV1aIGKm98c

53. https://avibarzeev.medium.com/for-xr-the-eyes-are-the-prize-25d43a533f2a 
metric. It's like asking smokers if adding flavor to their cigarettes makes them more or less enjoyable.

Some may argue that commercials are meant to inform us of products we might like. But more often ads attempt to create an emotional attachment to a brand or product that can't possibly deliver the promised emotion: love, belonging, happiness, or friendship. Consumers buy for emotional and social reasons, and not just because of information.

The real harm to consumers comes from ongoing manipulation by unaccountable forces that knowingly use people's private sensitivities against them. This may be the difference between interaction with a virtual dinosaur and having a digital twin of your loved one recommend you buy a sponsored item. Thus advertisement itself is not the problem, but rather the leveraging of personal or sensitive information about a user's body, mind, preferences, and behavior, in ways that are difficult for users to consent to-or even understand-and defend against.

The harm from collecting people's sensitive information and using it to target their emotional vulnerabilities is that an ad network can now customize its manipulation per person, even run rapid experiments on individuals to optimize the results. We'd each like to think we're too smart to be gamed, but everyone has emotional vulnerabilities. Intimacy requires vulnerability, and these highly personalized ads are essentially playing into this false intimacy.

With upcoming XR devices, the search for the best individual emotional triggers can be automated and validated by data collection of our behavior in the real world. Biometric sensors can gather information useful for ads, ${ }^{54}$ including our emotional responses to real and artificial stimuli, in ways that capitalize on our involuntary bodily reactions, like pupil dilation or skin moisture. Thus advertisements within XR may pose a particular problem related to consent that other advertisements do not share.

Companies that push these ads make more money by keeping users engaged for longer periods of time. Social networks, TV, and other ad-driven networks are designed to keep us engaged for as long as possible. XR ads are an opportunity to monetize the attention economy at a whole new level-in a fully-controlled and engaging environment. It's similar to a casino model, where consumers are the chips and the advertisers are making the bets on our behavior.

As a society, we are just beginning to come to grips with the ways that social network algorithms do harm to public discourse and mental health by feeding us more and more infuriating, unrealistic and radicalizing content to keep us hooked. Our dopamine receptors are engaged, our emotions enraged, in arguably addictive control patterns. Social media then behaves much like gambling or other psychological addictions. What happens if these impulses are combined-and augmented-by alternative reality that we treat as real? Advertisements in XR may end up feeling fundamentally more persuasive, and be less identifiable by users as sponsored content, and therefore it may be warranted to treat these experiences differently than the baseline FTC's "truth in advertising" standards. ${ }^{55}$

\section{Opportunities for Interventions and Further Research}

With increasing awareness of advertising in VR and AR, researchers, companies, and regulators will have opportunities for productive interventions, to mitigate the harms

54. https://scholarship.law.vanderbilt.edu/jetlaw/vol23/iss1/1/

55. https://www.ftc.gov/news-events/media-resources/truth-advertising 
listed above. There are also new areas to explore, given the possibilities of VR and AR to create virtual worlds, and the challenges brought by embodiment. If the brain is interpreting the virtual world as your actual reality, should that justify regulating the devices and their content, in ways that we do not limit other forms of advertising?

\section{Content moderation}

User generated content and organic interactions, as social media has demonstrated, may be rich in controversial content like harassment ${ }^{56}$ and hate speech. ${ }^{57}$ Social VR currently is grappling with how to moderate both content and behavior in immersive environments, which is more akin to a playground or a bar than a newsfeed or a tweet. How will companies moderate this content differently? Is it automated or will it require the equivalent of a digital hall monitor? Or will there be separate codes of conduct from web-based social networks, based on the different functionalities of XR mediums, including additional constraints on use and the addition of productive friction to prevent online harms?

We argue that, as a best practice, companies should not release social features in VR or AR before they have distinct terms of service, grounded in both neuroscience addressing immersive technology and research around social psychology, and specifically tailored for these unique digital spaces. More research should be done about how consumers interact and react to immersive content, including but not limited to VR and AR advertisements.

Immersive advertisements often take the form of gameplay and can include exciting or violent experiences. While these techniques can increase engagement, they raise important questions as to the impact they may have on users. Our brains trigger a visceral reaction to violence, so an ad that integrates a fighting element could have serious and long-term consequences. Our understanding of how we respond to these stimuli is woefully incomplete. For instance, one might think that using a cartoonish representation of violence would lessen its impact. But in some studies, cruder renderings in VR (for example, getting stabbed with a cartoon knife) actually seemed more realistic to users, because their brains filled in the display gaps at higher resolution than any headset. Therefore, platform policies to police the glorification of violence should not be evaluated in the same way that a newsfeed content moderator would evaluate a social media post, since the user's brain does not differentiate well between getting injured by an animated sword or a high-resolution scanned rapier-and being actually stabbed.

Furthermore, exactly who will be enforcing the rules and how users' actions and speech will be governed should also be made clear. This should take the form of procedural transparency, laying out how specific rules are applied to specific users' interactions. Users should be able to opt out of advertising and clearly know when content is paid or sponsored.

\section{Gig-Economy 2.0 for Content Creators}

What does an alternative business model to advertising in VR and AR practically look like? Most likely, it will incorporate e-commerce opportunities, including those for businesses to contract with XR content creators. But is this just another form of outsourced advertising?

56. https://www.extendedmind.io/the-extended-mind-blog/2018/04/04/2018-4-4-virtual-harassment-thesocial-experience-of-600-regular-virtual-reality-vrusers

57. https://www.adl.org/resources/reports/hate-in-social-virtual-reality 
User-generated XR content is not yet mainstream. Other than Roblox, Second Life and Minecraft, there are not yet simple and portable ways for most unskilled users to create their own immersive content or interactive virtual worlds. XR coding in Unity or Unreal Engine is a specialized skill. As such, VR has not had it's "YouTube moment." AR usergenerated content can still be considered to be nascent, as very few creators are able to make their creations into commercial successes.

There could be substantial benefits for increasing user-generated content as a monetization scheme. This may result in the democratization of content - just as YouTube and TikTok made content creation into a business that was accessible to anyone with a camera - and a greater diversity in the types of content created. More people will learn technical skills. Some will make money in The Metaverse, and some companies will profit off of the transactions, increased user base, and their audiences' creativity, as gaming companies have done before.

But there are also detriments. This model may replicate the exploitative harm of gig economies by creating a low cost for labor. A video investigation ${ }^{58}$ describes how Roblox benefits from child labor and unrealistic expectations of wealth and attention. What is stopping the same thing from happening with AR and VR creator marketplaces?

Additionally, cross-platform advertising risks creating more monopolies. Facebook collects some, but not all, of available data about users in its VR platform. ${ }^{59}$ But it still requires a Facebook account to log in. Consumers should question if that is because they already have access to enough classifiers for advertising-based purposes.

\section{New Business Models}

Surprisingly, if social networks were to shift to subscriptions and away from ads, their massive annual profit spread over their billions of users might come to only $\$ 20-\$ 50$ per person. ${ }^{60}$ If such companies put their present advertising energy into a new service working like Consumer Reports, objectively finding and rating consumer products, it would be a benefit to their newest customers: consumers. We've been sold on the idea that ads are required, but are they really?

Would breaking apart monolithic companies help avoid harms in XR? In the telecom world, AT\&T is notorious for its local monopolies on bandwidth, high prices and poor customer service. There's an old joke about what's better: fighting one horse-sized duck versus one-hundred duck-sized horses? What could happen when numerous smaller social networks have the same collective power and capitalize on the same business model as the biggest single networks do today?

Because of the way AR and VR interact with our neurology, and how the hardware requires involuntary stimuli to effectively function that could reveal private information about users, ${ }^{61}$ we question whether further propagating an ads-based model is responsible. Similarly, we would recommend an alternative business model be evaluated for its positive and negative aspects, including its impacts on the labor force, regardless of the size and influence of the company.

58. https://www.youtube.com/watch?v=_gXlauRB1EQ

59. https://www.roadtovr.com/oculus-quest-2-privacy-facebook-data-collection-settings/

60. https://www.forbes.com/sites/peggyannesalz/2017/08/15/how-much-are-users-really-willing-to-payfor-subscription-mobile-apps/?sh=784657354c69

61. https://scholarship.law.vanderbilt.edu/jetlaw/vol23/iss1/1/ 


\section{Privacy and Data Governance}

Digital identities are fundamental to questions about advertising and The Metaverse. How will your avatar be used in the future? Will your purchases and image be your own, or used to influence your social network in immersive spaces? What about issues of deepfakes and content substitution in advertising, in an environment designed to feel and look real, that may be personalized? Will what is addictive in social media and gaming prove even more harmful in VR and AR? Will there be a way to effectively opt-out of $\mathrm{XR}$ advertising, or will it be so pervasive that this would mean not participating in the digital ecosystem. What type of information, if any, will be cabined off by platforms and advertisers-and what type of information should be prohibited from advertising for the health of users and the health of the online ecosystem?

Right now we have more questions than answers, but it is important to consider these issues now, as the fundamental architecture of a shared Metaverse of immersive content is being created.

\section{Conclusion}

Advertising in VR and AR has always been a part of the ecosystem, even if it looks different than ads do in social media. This is not a cause for alarm in and of itself. We know immersive ads are more effective, but the real issue is how and why - and what are the implications of these questions. People enjoy the ads in XR because they are often experiential, but they risk being more manipulative and persuasive, based on the emotional and psychological aspects of immersive technology.

Additionally, because of the unique format of XR advertising, and how it blurs entertainment and persuasion, we need to consider questions about user privacy and cognition. How will XR advertising change the way consumers behave and interact with others-and what should be allowed or prohibited? Amidst all these questions, one thing is clear: if The Metaverse is only a conveyance of Facebook's family of apps, on pervasive and alternative interfaces to generate more ad revenue, it will be a failure of imagination. 


\section{Authors}

Brittan Heller is a human rights attorney with expertise in immersive technology, who is counsel in Global Business and Human Rights at Foley Hoag LLP. She is a non-residential fellow at the Atlantic Council focusing on the metaverse; was an inaugural AI and Technology Fellow at Harvard Kennedy School, and received the Nexant Prize for humanitarian service through VR from the Virtual World Society. Heller graduated from Stanford University and Yale Law School.

Avi Bar-Zeev has worked in Spatial Computing (AR/VR/MR/XR) for 30 years. He helped start the HoloLens at Microsoft and Echo Frames Amazon, and more recently helped Apple on undisclosed projects. He co-founded Keyhole, the startup that built Google Earth and helped define Second Life's core technology. Earlier, he worked on novel VR experiences for Disney. He's written extensively about invasive technologies, ways to prevent harm and empower individuals.

\section{Acknowledgements}

We thank Sara Bar-Zeev, Peter Alau, Nathaniel Gleicher, Mark Lemley, Helen Heller, Jennifer Bland, Jessica Outlaw, Alicia Wanless, Marco Di Miroz, Camille Francois, Christopher Lafayette, Kent Bye, Kate Klonick, and Ben Wittes for their support in developing these ideas and crafting the piece. Brittan Heller's employer's clients include technology companies and she has served as outside counsel and an external advisor to Facebook on privacy policy, human rights, and integrity-related issues. Avi Bar-Zeev does consulting on XR. 